\title{
The use and perception of support walkers for children with disabilities: a United Kingdom survey
}

\author{
Ciaran George ${ }^{1} \mathbb{D}$, Wendy Levin² and Jennifer M. Ryan ${ }^{1,3,4^{*}}$
}

\begin{abstract}
Background: Support walkers are a type of assistive device that may enable non-ambulant children with disabilities to walk independently and promote improvements in bowel function, bone mineral density (BMD), mobility, independence, participation and social function. However, there is little evidence to support these benefits and there is a lack of research describing the use of support walkers in clinical practice. This study aimed to examine the use of support walkers for children with disabilities in clinical practice.

Method: A survey was distributed via professional organisations, charities and schools associated with paediatric disabilities in the UK. Participants were recruited between January and March 2018. Populations of interest were those who prescribe support walkers to children with disabilities and those who work with children who use them.

Results: In total, 125 people were included in the analysis; 107 responders prescribed support walkers and 18 responders worked with children who used support walkers. The population of children who use support walkers ranged from 6 months to 18 years and included children with cerebral palsy, chromosomal abnormalities and other medical conditions. Use of these devices was also reported in schools, at home and in the community for varying lengths of time. Numerous perceived benefits were noted, most frequent of which were increases in physical activity and enjoyment. By comparison, fewer perceived problems were identified but centred on lack of space and difficulty with transfers.

Conclusions: This study provides insight into the use of support walkers in the UK, particularly surrounding current practices, which may help to improve consistency in clinical settings. Perceived benefits and problems may provide a basis for identification of appropriate outcome measures to monitor effectiveness. These results should also provide a basis for designing future studies to examine effectiveness of support walkers for paediatric disabilities.
\end{abstract}

Keywords: Paediatric, Support walker, Disability

Children with disabilities, particularly those with severe motor impairments, participate in less physical activity and exhibit more sedentary behaviour than their typically developing peers $[1,2]$. Barriers to participation in

\footnotetext{
* Correspondence: Jennifer.ryan@brunel.ac.uk

'College of Health, Medicine and Health Sciences, Brunel University London, London, UK

${ }^{3}$ Department of Public Health and Epidemiology, RCSI University of Medicine and Health Sciences, Dublin, Ireland

Full list of author information is available at the end of the article
}

physical activity include stigma, negative attitudes, lack of motor skills and inaccessible environments [3, 4]. Assistive devices are one method used to enable children to overcome these barriers and hence increase physical activity.

Handheld walkers are commonly used to promote independent locomotion. However, these may not be feasible for children with severe mobility impairments who lack the motor control or strength to stand upright [5].

(c) The Author(s). 2020 Open Access This article is licensed under a Creative Commons Attribution 4.0 International License, which permits use, sharing, adaptation, distribution and reproduction in any medium or format, as long as you give appropriate credit to the original author(s) and the source, provide a link to the Creative Commons licence, and indicate if changes were made. The images or other third party material in this article are included in the article's Creative Commons licence, unless indicated otherwise in a credit line to the material. If material is not included in the article's Creative Commons licence and your intended use is not permitted by statutory regulation or exceeds the permitted use, you will need to obtain permission directly from the copyright holder. To view a copy of this licence, visit http://creativecommons.org/licenses/by/4.0/ The Creative Commons Public Domain Dedication waiver (http://creativecommons.org/publicdomain/zero/1.0/) applies to the data made available in this article, unless otherwise stated in a credit line to the data. 
Support walkers are defined as any device that permits mobility for children who require more support than provided by handheld walkers, including some level of trunk, pelvic and/or head support [6]. Also known as 'gait trainers', there are many versions available with alterations in size, steering, seating and a range of accessories [6].

Support walkers may enable non-ambulant children the opportunity to walk independently. In a systematic review, several studies found positive changes across all dimensions of the International Classification of Functioning, Disability and Health [7], following use of support walkers including bowel function, bone mineral density (BMD), mobility, independence, participation and social function, with minimal adverse events [5]. However, the same systematic review suggested that the evidence was scarce, of low quality and inconsistent [5]. It was also noted that most of the current research focuses on children under 12 years of age with cerebral palsy [5], despite the potential of these devices in numerous other medical conditions [6]. Studies varied in terms of the protocol used and the outcomes assessed, thus limiting the conclusions that may be used to inform best practice.

While some studies have investigated the effectiveness of support walkers on a variety of outcomes, there is a lack of research examining how support walkers are currently used for children with disabilities in clinical practice. While one study has described the use of support walkers among children with disabilities in the United States [6], the study did not fully explore the perceived benefits, problems and reason to discontinue use of support walkers. Before further evaluating effectiveness of support walkers, information regarding the population who are prescribed support walkers, when and how they are used in routine clinical practice, and the perceived benefits and problems of using support walkers, is needed [8]. As such, this study aimed to examine the use of support walkers for children with disabilities in clinical practice. Specific questions were: (1) Which children use support walkers?; (2) Which support walkers are prescribed and which factors affect this decision?; (3) How are support walkers used?; (4) What are the perceived benefits and problems associated with using support walkers?

\section{Methodology}

\section{Design}

A cross-sectional survey design was used. Approval for the current research study was granted by Brunel University London Research Ethics Committee (Reference: 7627-A-Jan/2018-10,863-1). Completion of the survey indicated consent. All responses were anonymous.

\section{Participants}

Participants were recruited over 10 weeks between January and March 2018. Professionals who prescribe support walkers, such as physiotherapists (i.e., prescribers), and those who work with children who use support walkers but do not prescribe them, such as teachers (i.e., non-prescribers) were invited to complete a survey. To be included in the study, prescribers must have prescribed a support walker to at least one child, aged $0-18$ years. To be included in the study, nonprescribers must have worked with at least one child, aged 0-18 years who used, or currently uses a support walker. A support walker was defined as any device that permits mobility for children who require more support than provided by handheld walkers, including some level of trunk, pelvic and/or head support [6]. No restrictions were placed on type of disability due to the exploratory nature of this study.

Information about the study was shared with 33 Special Educational Needs (SEN) schools, 9 professional organisations and 10 charities for young people with disabilities in the UK. These were identified via an internet search. Emails were sent and replies were received from eight UK organisations and charities for children with disabilities (e.g., the Association of Paediatric Chartered Physiotherapists) and eight SEN schools. Gatekeepers who worked in each organisation (e.g. an administrator, a physiotherapist, a principal) distributed a brief description of the study and link to the information sheet and survey via email or social media. One school was provided with paper versions of the survey and information sheet to distribute at their request.

\section{Data collection}

Separate surveys were developed for prescribers and non-prescribers following a literature review [5, 6, 9-12] and discussion with an experienced paediatric physiotherapist who prescribes support walkers. Both surveys included questions relating to characteristics of the children, normal use of the devices, and the perceived benefits and problems associated with their use. The penultimate draft of each survey was reviewed by an advisory panel, consisting of a physiotherapist and two teachers working in a SEN school, who assessed the questions for ease of interpretation, accuracy and comprehensiveness. Input from this panel prompted the following changes: the addition of responses to multiple questions, rephrasing of several questions, removal of questions that added confusion, and the addition of questions that required further expansion. At the start of each survey, participants were provided with the definition of a support walker, as stated previously, with 3 typical examples of support walkers. 
The surveys contained 28 and 29 questions for the non-prescriber and prescriber surveys respectively, taking approximately $10-15 \mathrm{~min}$ to complete. The terms 'Please tick all that apply' or 'Please select only one response' followed the questions to maximise clarity. Most questions were close-ended, although there were three open-ended questions to permit greater exploration of opinions and depth of understanding. The open-ended questions were: "What, in your opinion, would be the ideal age to begin use of a support walker?"; "Please identify if there are any precautions for use of support walkers (e.g. supervision)" (Only present in the prescribers survey); "Any other comments?"

\section{Data analysis}

Prior to data analysis, surveys in which responders reported working exclusively with adults (over 18 years old) were excluded. Descriptive statistics (mean, SD, range and percentages) were used to report data. If five or more participants provided the same response to a question where there was an option to select "other", these responses formed a new category. Where a range of ages was given in response to the 'age of introduction' questions, the mean of this range was calculated and subsequently included in the calculation for mean of all responders. Responses to open questions were analysed using a simplistic form of content analysis, whereby similar responses were assigned a code and percentages of each code were calculated. Responses to the 'any other comments' question underwent a more detailed process of content analysis as described by Elo and Kyngäs [13], whereby important phrases were noted, matched to similar phrases and subsequently grouped into categories. The final stage, 'interpretation', was removed from this content analysis since the nature of inquiry did not permit knowledge of context or the ability to probe participants [14].

\section{Results}

One hundred twenty-six surveys were returned; 107 from prescribers and 19 from non-prescribers. One nonprescriber survey was excluded as the responder reported only working with adults; thus, 18 were included in analysis. No response rates could be calculated as it was unknown how many eligible individuals received the survey.

\section{Responder demographics}

Most prescribers were female (97.2\%) and physiotherapists $(98.1 \%)$. One occupational therapist and one therapy assistant also reported prescribing support walkers. Prescribers reported working across multiple settings including the community setting (80.4\%), SEN schools (57.9\%), mainstream schools (42.1\%), outpatient settings
(28.0\%), inpatient settings (6.5\%) and 'other' areas (3.7\%). The occupation of non-prescribing professionals was more heterogeneous, comprising of physiotherapists (33.3\%), occupational therapists (33.3\%), teachers (22.2\%), classroom assistants (5.6\%) and other (5.6\%). Prescribers and non-prescribers had worked with children using support walkers for a mean of 16.1 (range 2-35 years), and 11.7 (range 1-25 years) years, respectively.

\section{Characteristics of the children}

Table 1 outlines the characteristics of children using support walkers as reported by prescribers and nonprescribers. Support walkers were most commonly reported to be prescribed and used by children between 2 and 12 years (Table 1 ). However, support walkers were prescribed and used by children of all ages ( $0-18$ years), with three prescribers $(2.8 \%)$ prescribing support walkers to children between 0 and 1 years (Table 1). Prescribers reported that the mean (standard deviation [SD]) age of introduction to support walkers was 3.6 (1.6) years. The youngest age of introduction, however, was reported by prescribers and non-prescribers as a mean (SD) of 2.4 (1.4) years (range; $1-14$ years) and 2.9 (1.0) years (range; 1-5 years), respectively. When asked at what age children should ideally be introduced to support walkers, 77 prescribers responded giving a mean (SD) age of 2.5 (1.0) years (range 1-6 years). Thirty prescribers provided text responses which revealed that ideal age of introduction was dependent on the child's condition $(n=20$, $66.7 \%)$, more specifically their stage of development, cognition, motivation and motor ability. Five responders (16.7\%) reported support walkers were often prescribed following recognition that a child was attempting to weight bear and step.

All prescribers reported prescribing support walkers to children with spastic cerebral palsy. Many prescribers also reported prescribing support walkers to children with dyskinetic $\mathrm{CP}$, ataxic $\mathrm{CP}$, chromosomal abnormalities and spina bifida (Table 1). Similarly, the majority of non-prescribers reported that children with spastic $\mathrm{CP}$, dyskinetic CP, ataxic CP and chromosomal abnormalities used support walkers (Table 1). However, only $16.7 \%$ of non-prescribers reported that children with spina bifida used support walkers compared to $52.3 \%$ of prescribers (Table 1 ).

Approximately $90 \%$ of prescribers and non-prescribers reported that children using support walkers also used a wheelchair (Table 1). However, $20.6 \%$ of prescribers reported that children who used a support walker can walk with a handheld walker and $15.0 \%$ of prescribers reported that children who used a support walker can walk independently (Table 1). 
Table 1 Characteristics of the children who use support walkers as reported by prescribers and non-prescribers

\begin{tabular}{|c|c|c|}
\hline & $\begin{array}{l}\text { Prescribers }(n=107) \\
n(\%)\end{array}$ & $\begin{array}{l}\text { Non-prescribers }(n=18) \\
n(\%)\end{array}$ \\
\hline \multicolumn{3}{|l|}{ Age groups } \\
\hline $0-1$ years & $3(2.8)$ & $2(11.1)$ \\
\hline $1-2$ years & $57(53.3)$ & $3(16.7)$ \\
\hline $2-5$ years & $95(88.8)$ & $13(72.2)$ \\
\hline $5-12$ years & $93(86.9)$ & $14(77.8)$ \\
\hline $12-16$ years & $77(72.0)$ & $11(61.1)$ \\
\hline 16-18 years & $48(44.9)$ & $7(38.9)$ \\
\hline \multicolumn{3}{|l|}{ Medical Diagnosis } \\
\hline Spastic CP & $107(100)$ & $17(94.4)$ \\
\hline Dyskinetic CP & $90(84.1)$ & $13(72.2)$ \\
\hline Ataxic CP & $78(72.9)$ & $12(66.7)$ \\
\hline Chromosomal Abnormalities & $83(77.6)$ & $9(50.0)$ \\
\hline Spina bifida & $56(52.3)$ & $3(16.7)$ \\
\hline Muscular Dystrophy & $53(49.5)$ & $6(33.3)$ \\
\hline Rett syndrome & $49(45.8)$ & $3(16.7)$ \\
\hline Epilepsy & $46(43.0)$ & $10(55.6)$ \\
\hline Spinal Cord Injury & $41(38.3)$ & $2(11.1)$ \\
\hline Developmental Delay ${ }^{a}$ & $16(15.0)$ & - \\
\hline Other & $15(14.0)$ & $1(5.6)$ \\
\hline \multicolumn{3}{|l|}{ Alternative form of mobility } \\
\hline Wheelchair & $98(91.6)$ & $16(88.9)$ \\
\hline Handheld walker & $22(20.6)$ & $8(44.4)$ \\
\hline Nothing (independent walker) & $16(15.0)$ & $2(11.1)$ \\
\hline Adult facilitated $^{\mathrm{a}}$ & $6(5.6)$ & - \\
\hline Other & $12(11.2)$ & $5(27.8)$ \\
\hline
\end{tabular}

Dash (-) indicates the items that were unavailable to that population

arepresents a category that was created when enough responders reported it within the 'other' option

\section{Prescription of support walkers}

A child's level of mobility was the most common factor affecting prescription of support walkers, as reported by 97.2\% of prescribers (Table 2). A child's tolerance of the support walker and current activity level were the second and third most reported determinants of support walker prescription (Table 2). Many prescribers noted that lack of head control (78.5\%), pain (74.8\%), and behavioural issues $(57.0 \%)$ were contraindications to the prescription of support walkers (Table 2). Eighty percent of prescribers reported that the needs of the child affected the duration of use they prescribed, followed by clinical experience (76.6\%), goals of physiotherapy $(59.8 \%)$, evidence $(26.2 \%)$, and local or national guidelines (9.3\%) (Supplemental Table 1).

In total, 21 support walkers were identified by prescribers (Supplemental Table 2). Most commonly prescribed support walkers were the Rifton Pacer (88.8\%), Ormesa Grillo (39.3\%) and Buddy Roamer (35.5\%)
(Supplemental Table 2). When choosing which support walker to prescribe, prescribers most commonly reported that clinical experience (91.6\%), needs of the child $(89.7 \%)$ and goals of physiotherapy (72.9\%) affected their decision (Supplemental Table 3). The inclusion of parents (98.1\%) and the child (82.2\%) within this decision was also reported by most prescribers (Supplemental Table 3).

\section{Use of support walkers}

The environments in which prescribers reported support walkers are used included: at home (88.8\%), in schools (94.4\%) and in the community (69.2\%) (Supplemental Table 4). Similarly, non-prescribers reported support walkers were used in schools $(100 \%)$, at home $(72.2 \%)$ and in the community (38.9\%) (Supplemental Table 4).

When asked how long prescribers would recommend using the support walker per day, $49.5 \%$ suggested 'as much as able' (Supplemental Table 5). Others 
Table 2 Responses from prescribers regarding the factors affecting prescription and common contraindications Factors influencing prescription of support walkers

A child's mobility level

A child's tolerance of the support walker

The activity level of the child

A child's enjoyment

A child's motor control

Gait pattern

Family support

Space to use and store the support walker

A child's cognitive status

Muscle weakness

Medical needs of the child

A child's balance

Cost of the support walker

Available evidence

Child's aerobic endurance

Type of school a child attends

Other

Common contraindications to the use of support walkers

Prescribers $(\boldsymbol{n}=\mathbf{1 0 7})$
$\boldsymbol{N}(\%)$
$84(78.5)$
$80(74.8)$
$61(57.0)$
$39(36.4)$
$35(32.7)$
$32(29.9)$
$23(21.5)$
$22(20.6)$
$19(17.8)$
$18(16.8)$
$17(15.9)$
$16(15.0)$
$12(11.2)$
$11(10.3)$
$7(6.5)$

Lack of head control

Pain experienced in the walker

Behaviour issues

Current hip dislocation

Cognitive impairment

Muscle contractures

Skeletal deformities

The weight of the child

Lack of trunk control

Visual impairment

Epilepsy

Reduced bone mineral density

The height of the child

Risk of hip dislocation

Other

recommended either: $10-30 \mathrm{~min}$ (5.6\%); 30-60 min (31.8\%), 1-2 h (11.2\%) or 2-5 h (1.9\%) (Supplemental Table 5). In contrast, non-prescribers reported that support walkers were used for less than $10 \mathrm{~min}$ (5.9\%), 10$30 \mathrm{~min}$ (35.3\%), 30-60 min (35.3\%), 1-2 h (17.6\%) or all day (5.9\%) (Supplemental Table 5). The perceived reasons for discrepancies between prescribed and actual use are given in Table 3. Most prescribers suggested a lack of staff to help the child (88.8\%) and/or a lack of space to use the walker (72.9\%) prevented use (Table 3). However, among non-prescribers, 39\% did not know which factors prevented the duration being achieved and 39\% reported that the child simply preferred another means of mobility (Table 3 ).

Ninety prescribers (84.1\%) provided a response to question "Please identify if there are any precautions for use of support walkers". The requirement for supervision was most commonly reported $(n=73,68.2 \%)$. The 
Table 3 Reasons why 'actual' duration may be less than the prescribed duration

\begin{tabular}{lll}
\hline & Prescribers $(\boldsymbol{n}=\mathbf{1 0 7}), \boldsymbol{N}(\%)$ & Non-prescribers $(\boldsymbol{n}=\mathbf{1 8}), \boldsymbol{N}(\mathbf{\%})$ \\
\hline Lack of staff to help the child & $95(88.8)$ & $6(33.3)$ \\
Lack of space to use the walker & $78(72.9)$ & $4(22.2)$ \\
Child prefers other means of mobility & $36(33.6)$ & $7(38.9)$ \\
The walker causes the child pain & $19(17.8)$ & $2(11.1)$ \\
The child doesn't like the look & $4(3.7)$ & $0(0.0)$ \\
Boredom & $20(18.7)$ & $2(11.1)$ \\
The walker is uncomfortable to use & $22(20.6)$ & $0(0.0)$ \\
Doesn't like different position & $8(7.5)$ & $0(0.0)$ \\
They are shared between children & $16(15.0)$ & $4(22.2)$ \\
Fatigue & $6(5.6)$ & - \\
Other & $15(14.0)$ & $3(16.7)$ \\
Don't know & - & $7(38.9)$ \\
\hline
\end{tabular}

Dash (-) indicates the items that were unavailable to that population

arepresents a category that was created when 5 or more similar responses were given in the 'other' option

second most frequently reported precaution was the need for a suitable environment including a suitable surface, adequate space and distance from other children ( $n=48 ; 53.3 \%)$. Other precautions to consider were the need for competent or trained staff and parents $(n=25$; $27.8 \%)$ and the presence of other medical conditions, particularly those with epilepsy $(n=8 ; 8.9 \%)$.

Before use of these devices is discontinued by a child, $42.1 \%$ of prescribers reported that they are used for 510 years (Supplemental Table 6). Others reported that they were used for less than 1 year (1.9\%), 1-2 years (2.8\%), 2-5 years (34.6\%), and over 10 years (18.7\%) (Supplemental Table 6). Non prescribers reported use for 2-5 years (50\%), 5-10 years (27.8\%), over 10 years (11.1\%), $1-2$ years $(5.6 \%)$ or less than 1 year $(5.6 \%)$ (Supplemental Table 6).

Approximately $75 \%$ of prescribers suggested that between 0 and $20 \%$ of children who use support walkers show progression in walking ability, such that they no longer require the support walker (Supplemental Table 7). When asked what affects a child's chance of walking progression, most prescribers reported that it was due to: the child's condition (96.3\%), original level of head or trunk control (79.4\%), frequency of use $(51.4 \%)$, age of introduction (21.5\%), motivation (10.3\%) and cognitive ability (6.5\%) (Supplemental Table 7).

Table 4 reports the reasons children discontinue use of their support walker. Progression of their condition, such that they are no longer able to use support walkers was most frequently reported by prescribers (79.4\%) and non-prescribers (72.2\%) (Table 4). Both groups also reported that discontinuation occurred because children prefer other means of mobility or progress their walking ability (Table 4).

Table 4 Responses given for the reason children discontinue using support walkers

\begin{tabular}{lll}
\hline & Prescribers $(\boldsymbol{n}=\mathbf{1 0 7}), \boldsymbol{N}(\%)$ & Non-prescribers $(\boldsymbol{n}=\mathbf{1 8}), \boldsymbol{N}(\mathbf{\%})$ \\
\hline Progression of condition (i.e. unable to use the SW) & $85(79.4)$ & $13(72.2)$ \\
Other means of mobility are preferred & $50(46.7)$ & $11(61.1)$ \\
Progression of walking ability & $46(43.0)$ & $9(50.0)$ \\
Frustration with the SW & $40(37.4)$ & $7(38.9)$ \\
Not enough space to use the SW & $37(34.6)$ & $5(27.8)$ \\
Safety reasons regarding the size of the child & $36(33.6)$ & $6(33.3)$ \\
Fractures or other medical complications & $25(23.4)$ & $4(22.2)$ \\
Other & $12(11.2)$ & $1(5.6)$ \\
Left the school that provided the SW & $8(7.5)$ & $2(11.1)$ \\
Negative social perceptions & $6(5.6)$ & $1(5.6)$ \\
Boredom & $6(5.6)$ & $2(11.1)$ \\
\hline
\end{tabular}


Table 5 Perceived Benefits of using support walkers as perceived by all responders

\begin{tabular}{|c|c|c|}
\hline & $\begin{array}{l}\text { Prescribers }(n=107) \\
n(\%)\end{array}$ & $\begin{array}{l}\text { Non-Prescribers }(n=18) \\
n(\%)\end{array}$ \\
\hline Increase time being physically active & $105(98.1)$ & $17(94.4)$ \\
\hline Enjoyment & $101(94.4)$ & $15(83.3)$ \\
\hline Increase participation in everyday life & $97(90.7)$ & $15(83.3)$ \\
\hline Provides different opportunities to access their environment & $95(88.8)$ & $13(72.2)$ \\
\hline Increase independence & $92(86.0)$ & $17(94.4)$ \\
\hline Increased confidence & $91(85.0)$ & $13(72.2)$ \\
\hline Improved muscle strength & $91(85.0)$ & $14(77.8)$ \\
\hline Provides a change of position & $91(85.0)$ & $14(77.8)$ \\
\hline Improved motor abilities (i.e. walking) & $89(83.2)$ & $12(66.7)$ \\
\hline Increased peer and family interaction & $89(83.2)$ & $14(77.8)$ \\
\hline Improved respiratory function & $73(68.2)$ & $8(44.4)$ \\
\hline Increase bone mineral density & $67(62.6)$ & $9(50.0)$ \\
\hline Improved head and trunk control & $65(60.7)$ & $8(44.4)$ \\
\hline Improved bladder and bowel function & $58(54.2)$ & $8(44.4)$ \\
\hline Improved problem solving (i.e. navigation) & $54(50.5)$ & $9(50.0)$ \\
\hline Prevents muscle wasting & $50(46.7)$ & $8(44.4)$ \\
\hline Improved communication & $36(33.6)$ & $7(38.9)$ \\
\hline Improved cognition & $22(20.6)$ & $3(16.7)$ \\
\hline Improved vision & $9(8.4)$ & $3(16.7)$ \\
\hline Other & $4(3.7)$ & $2(11.1)$ \\
\hline
\end{tabular}

\section{Perceived benefits and problems}

Table 5 shows the perceived benefits of using support walkers. The three most reported perceived benefits by prescribers were increasing physical activity time (98.1\%), enjoyment (94.4\%), and increasing participation (90.7\%). Increased independence (94.4\%), increased time being physically active (94.4\%), and enjoyment (83.3\%) were most reported perceived benefits by non- prescribers (Table 5). However, many more perceived benefits were reported by over half of prescribers and non-prescribers, including improved muscle strength, improved motor abilities, increased peer and family interaction, improved respiratory function and increased bone mineral density (Table 5).

The perceived problems experienced with support walkers are shown in Table 6. Lack of space was the

Table 6 Perceived problems with support walkers as perceived by all responders

\begin{tabular}{lll}
\hline & Prescribers $(\boldsymbol{n}=\mathbf{1 0 7}), \boldsymbol{N}(\%)$ & Non-prescribers $(\boldsymbol{n}=\mathbf{1 8}), \boldsymbol{N}(\mathbf{\%})$ \\
\hline Not enough space & $85(79.4)$ & $10(55.6)$ \\
Getting the child in or out of the SW & $72(67.3)$ & $9(50.0)$ \\
Lack of accessibility & $69(64.5)$ & $10(55.6)$ \\
The time required to transfer in and out of the SW & $50(46.7)$ & $3(16.7)$ \\
Trouble moving on difference surfaces & $47(43.9)$ & $10(55.6)$ \\
Time to use the SW & $45(42.1)$ & $4(22.2)$ \\
Poor manoeuvrability & $37(34.6)$ & $12(66.7)$ \\
Lack of knowledge about SW & $21(19.6)$ & $3(16.7)$ \\
Problems adjusting the walker if shared & $16(15.0)$ & $2(11.1)$ \\
Risk of tipping & $12(11.2)$ & $5(27.8)$ \\
Negative social perceptions & $8(7.5)$ & $3(16.7)$ \\
Other & $7(6.5)$ & $2(11.1)$ \\
\hline
\end{tabular}


most commonly reported problem among prescribers (79.4\%) (Table 6). Other perceived problems reported by prescribers included: getting in or out of the walker (67.3\%), and lack of accessibility (64.5\%) (Table 6). Poor manoeuvrability was the most commonly reported problem perceived by non-prescribers (66.7\%) (Table 6).

\section{'Any other comments?'}

Thirty-three participants (29 prescribers and 4 nonprescribers) provided a response to the question 'any other comments'. Where quotations are reported below, the responder's individual identification (ID) number follows.

\section{Category 1: the age to introduce support walker}

This category details two conflicting points of view regarding the age that support walkers should be introduced. The first is that support walkers should be introduced early as this may precipitate independent walking. Conversely, others believed that support walkers should only be introduced after no further gain in walking ability is expected. The contrasting points of view are exemplified below.

"we strive to get children walking as soon as they tolerate supported standing or start nursery when peers are exploring areas in standing ... many go on to independent walking without aids. An early approach to walking puts steps in place for the child to progress quicker and reduced frustration at not being able to get about." (Participant 38)

"[I] generally don't prescribe them until later when it becomes evident that the child's walking potential has been met and only then for enjoyment and [the] other factors mentioned" (Participant 17)

\section{Category 2: lack of funding}

Comments highlighting issues acquiring funding for support walkers were common. For example,

"it is frustrating that they mostly have to be privately funded or by seeking charity funds" (Participant 103)

Some prescribers also noted that even when funding was available certain criteria must be met, such that they may only be prescribed if the child is expected to gain the ability to walk, as in the next quote.

"our manager will not allow NHS funding for a supportive walker unless it is likely to lead to that child becoming an independent walker" (Participant 99)

\section{Category 3: perceived benefits}

Several prescribers remarked that support walkers are primarily for non-ambulant children, although a wide range of children may use them. The quotations below exemplify this:

"In my experience walkers give children with otherwise no independent mobility a change of position, exercise and mobility which they love" (Participant 57)

"Some early [developmental] delay children we also put in as a way of gaining weight bearing but they will not be long term users and progress to independent walking." (Participant 89)

\section{Discussion}

This study aimed to examine the use of support walkers for children with disabilities in clinical practice in the UK. The findings highlight that support walkers are prescribed to children with a variety of medical conditions including cerebral palsy, chromosomal abnormalities and spina bifida. Mobility level, tolerance and activity level of the child were the most reported factors affecting prescription of support walkers which were subsequently used for differing lengths of time per day. Many perceived benefits, including increasing physical activity, enjoyment and participation were reported. A substantial gap in the literature on support walkers exists, making comparisons to previous research difficult.

Below is a discussion of the findings in relation to each research question.

\section{Which children use support walkers?}

While prescribers and non-prescribers report that most children who use support walkers have cerebral palsy, these findings highlight that support walkers are prescribed to children with a substantial diversity of conditions. Children with chromosomal abnormalities were the third most frequently reported group that support walkers were prescribed to, even though no previous research examining the effects of support walkers have included this population.

This study found that some children are prescribed support walkers from as young as one-year-old. However, children below the age of $3 \frac{1}{2}$ years old are frequently excluded from participating in studies examining effectiveness of support walkers $[9,10]$. Although, an average of $2^{1 / 2}$ years old was deemed 'ideal', this is far behind the 12.1 months at which typically developing children begin walking independently [15]. It may be that earlier introduction encourages motor development and consequently psychological and cognitive development [16]. It was clear that earlier use was 
encouraged by some prescribers who noted a potential to progress to less supportive walking aids as development occurs. However, contrary to that opinion, others indicated that they would introduce support walkers only when no further improvement in walking was seen. These diverse opinions among prescribers may explain why there was substantial variation in responses from prescribers with regards to the "ideal" age at which to introduce support walkers, with a range between 1 and 5 years. Many responders reported that the 'ideal' age is specific to the individual and depends upon the needs of the child. It may be that differences in the perceived benefits of support walkers among prescribers may contribute to diversity of opinion regarding the age at which to introduce them.

\section{Which support walkers are prescribed and which factors affect this decision?}

Several types of support walkers were reportedly used, most of which currently have no research investigating their effectiveness. However, approximately a quarter of responders suggested that the available evidence influenced their decision. A child's mobility level and motor control were commonly reported to influence prescription of support walkers. Previous research examining effectiveness has commonly excluded children of higher motor function $[9,11]$. However, prescribers in this study reported that support walkers may be used as a bridge to independent walking, with improved walking ability being a reason for discontinuation. This is in agreement with findings from previous studies $[11,17]$.

\section{How are support walkers used?}

Most responders reported that children continue using support walkers for 5-10 years, far exceeding the duration of follow-up in previous research, which ranged from 3 months to 3 years $[6,9-11]$. Although interventions imposed time restrictions for obvious reasons, Low et al. [6] indicated that they are most commonly used for less than 6 months. Since no explanation was given by Low et al. [6] for discontinuation, it can only be speculated that there exists differences in the prescription and use of support walkers between the UK and USA.

Deterioration of a child's condition was identified in this study as the most reported reason for discontinuation. The progressive nature of some conditions (e.g. muscular dystrophy) or negative changes that occur during growth spurts (e.g. scoliosis and hip problems) will likely make walking difficult or impossible for these children [18]. The resulting pain or increased energy requirements may explain why a preference for another form of mobility (e.g. wheelchairs) may subsequently arise [19].
Almost half of prescribers reported support walkers should be used as much as able, suggesting that longer use is encouraged. However, both prescribers and nonprescribers also noted that a lack of staff limited the duration of use. Huang et al. [20] remarked that teachers are rarely able to encourage the use of assistive devices, as they are preoccupied with large class sizes and academic success of every child. As such, the requirement for supervision, as noted by the majority of responders, is likely unfeasible in most educational settings including SEN provision, at least for a prolonged period. Lack of space was also noted to affect how long support walkers are used, as well as represent a precaution to be considered when prescribing support walkers.

\section{What are the perceived benefits and problems associated with using support walkers?}

Responders perceived that support walkers have numerous benefits. The most commonly reported perceived benefits were increased physical activity, participation and enjoyment. Fewer prescribers and non-prescribers reported physiological changes associated with use of support walkers, including bone mineral density and bladder and bowel function. Despite never being examined as an outcome in studies of effectiveness of support walkers, increasing the amount of time a child is physically active was the most reported benefit by prescribers and non-prescribers. For a group that commonly exhibits less physical activity, support walkers may therefore promote participation in physical activity and associated health benefits, such as reduced pain, fatigue and osteoporosis among children with disabilities [21]. In addition, partaking in physical activity may explain subsequent increases in confidence and self-esteem, which were noted as perceived benefits by the majority of responders and in previous research [12, 22].

The perception that support walkers increased participation in everyday life for children with disabilities was commonly reported. This may relate to many tasks encountered during the typical day, from education to play. Being upright affords children different opportunities to experience their surroundings, which was frequently reported in this research. McKeever et al. [12] found that parents thought an altered perception of the environment, which arose from being upright, increases participation, as well as interaction with peers and family members. Social function, however, showed no change in two previous studies of the effects of support walkers $[9,10]$. Researchers have concluded that outcome measures available to assess participation may not adequately do so, which may explain the lack of support beyond these subjective accounts [23]. In addition to potential benefits on development and health, participation in leisure activities and greater enjoyment has been 
shown to increase a child's quality of life (QOL) [24]. However, the effect of using support walkers on quality of life has not been examined and should be considered as an outcome in future studies of effectiveness.

Lack of space was perceived as a problem by many prescribers and non-prescribers in this study. Huang et al. [25] reported that a lack of space to move around or store big devices, like support walkers, led many children to abandon use of assistive devices at home. As one of the biggest assistive devices, it is not difficult to imagine why space, manoeuvrability and accessibility were frequently reported issues. Many prescribers and nonprescribers also perceived that transferring a child to the support walker, and the time required to do so, was a problem when using support walkers.

Instability and reports of support walkers tipping over were noted in previous literature when using a particular support walker (David Hart walking orthosis), particularly when on uneven surfaces outside [10]. Almost half of the prescribers and non-prescribers in this study also stated that using support walkers on different surfaces was problematic. Despite this, use in the community was reported by over half of prescribers and non-prescribers. Hence, rather than limiting use, the requirement for constant supervision, which was noted as a precaution by prescribers, may mitigate these risks.

\section{Limitations}

A relatively small sample size and the inability to calculate response rates are perhaps the most important limitations associated with this study, especially for the nonprescribing group. The sample of convenience may have also introduced selection bias. In addition, despite the use of open-ended questions, the survey design prevented the ability to probe responders and thereby lack the conceptual richness and depth of knowledge. This study is also limited in that these results only provide a snapshot and do not adequately describe the process of prescription.

This study describes the paediatric populations who use support walkers, which may enable clinicians to consider their current practice, especially regarding the use of support walkers among children with medical conditions, such as chromosomal abnormalities, where no previous research exists. Identification of common contraindications or precautions for use may also support the clinical decision process when prescribing support walkers. Additionally, identification of common problems may enable individuals to put in place strategies to overcome these common barriers before prescribing support walkers. Prescribers should also take note of the perceived benefits, as they may be used to support an individual's decision to use support walkers as well as provide a list from which appropriate outcome measures may be selected to monitor progress. The findings from this study may also provide a basis for future research. Randomised controlled trials are needed to establish the effectiveness of support walkers. These findings may support researchers to define the population, identify appropriate outcomes, and determine an appropriate duration of follow-up in future trials. Investigation of differences in outcomes between age groups or conditions may also promote clearer decisions surrounding the prescription of support walkers.

\section{Conclusion}

In summary, this study provides an insight into the current use and perception of support walkers for children with disabilities in the UK. Findings suggest that the population of children that use support walkers is far more diverse than previously included in studies of effectiveness. There is large variation in current clinical practice, including the age at which support walkers are prescribed, the functional mobility of children who are prescribed support walkers, and the duration for which they are used. Numerous perceived benefits were reported, including increased participation, physical activity and enjoyment. The effectiveness of support walkers on many of these outcomes has not been previously examined. These findings should represent the first step to improve consistency in clinical practice and aid the clinical decision-making process. However, further research, with these findings in mind, must be conducted so that clinicians may deliver evidence-based practice.

\section{Supplementary Information}

The online version contains supplementary material available at https://doi. org/10.1186/s12887-020-02401-5.

\footnotetext{
Additional file 1: Supplemental Table 1. Prescribers reasoning behind a given duration of use. Supplemental Table 2. Types of support walkers used among prescribers and non-prescribers. Supplemental Table 3. Responses from prescribers regarding the factors that influence which support walker is prescribed and the individuals involved in this decision. Supplemental Table 4. The environments in which support walkers are used, as reported by prescribers and non-prescribers. Supplemental Table 5. Prescribed and actual daily duration of support walker use, as reported by prescribers and non-prescribers. Supplemental Table 6. The duration support walkers are used for within a child's lifespan, as reported by prescribers and non-prescribers. Supplemental Table 7. Percentage of children for whom their walking ability progresses (e.g. handheld or independent walking) and the factors which affect this.
}

Additional file 2. Example of a support walker

\section{Abbreviations}

QOL: Quality of life; SEN: Special Educational Needs; GMFCS: Gross motor function classification system; UK: United Kingdom; USA: United states of America; NHS: National Health Service; SW: Support walker; CP: Cerebral Palsy; SD: Standard Deviation; BMD: Bone mineral Density 


\section{Acknowledgements}

Many thanks to the gatekeepers who assisted in the distribution of the surveys.

\section{Authors' contributions}

CG designed the work, acquired, analysed, and interpreted the data and drafted and revised the manuscript. JR conceived the work, interpreted the data and revised the manuscript. WL conceived the work, contributed to the design of work, contributed to data acquisition and revised the manuscript. All authors approved the final version.

\section{Funding}

No funding was provided to undertake this research.

\section{Availability of data and materials}

The dataset analysed during the current study is available from the corresponding author on reasonable request.

\section{Ethics approval and consent to participate}

Approval for the current research study was granted by Brunel University London Research Ethics Committee (Reference: 7627-A-Jan/2018-10863-1). Completion of the survey indicated consent.

\section{Consent for publication}

The participant information sheet made all participants aware that results will be published in a scientific journal.

\section{Competing interests}

The authors declare that they have no competing interests.

\section{Author details}

'College of Health, Medicine and Health Sciences, Brunel University London, London, UK. ${ }^{2}$ Paediatric Physiotherapy Department, Royal Free London NHS Foundation Trust, London, UK. ${ }^{3}$ Department of Public Health and Epidemiology, RCSI University of Medicine and Health Sciences, Dublin, Ireland. ${ }^{4}$ Mary Seacole Building, Brunel University London, Kingston Lane, London, Uxbridge UB83PH UK.

Received: 24 January 2020 Accepted: 22 October 2020

Published online: 18 November 2020

\section{References}

1. Maher CA, Williams MT, Olds T, Lane AE. Physical and sedentary activity in adolescents with cerebral palsy. Dev Med Child Neurol. 2007;49(6):450-7.

2. Steele CA, Kalnins IV, Rossen BE, Biggar DW, Bortolussi JA, Jutai JW. Agerelated health risk behaviors of adolescents with physical disabilities. Sozialund Praventivmedizin. 2004;49(2):132-41.

3. Shields N, Synnot AJ, Barr M. Perceived barriers and facilitators to physical activity for children with disability: a systematic review. Br J Sports Med. 2012;46(14):989-97.

4. Bloemen MAT, Backx FJG, Takken T, Wittink H, Benner J, Mollema J, de Groot JF. Factors associated with physical activity in children and adolescents with a physical disability: a systematic review. Dev Med Child Neurol. 2015;57(2):137-48.

5. Paleg G, Livingstone R. Outcomes of gait trainer use in home and school settings for children with motor impairments: a systematic review. Clin Rehabil. 2015;29(11):1077-91.

6. Low SA, McCoy SW, Beling J, Adams J. Pediatric physical therapists' use of support walkers for children with disabilities: a nationwide survey. Pediatr Phys Ther. 2011;23(4):381-9.

7. World Health Organization: International Classification of Functioning, Disability and Health (ICF). Geneva: World Health Organization; 2001.

8. Craig P, Dieppe P, Macintyre S, Michie S, Nazareth I, Petticrew M. Developing and evaluating complex interventions: the new medical research council guidance. Int J Nurs Stud. 2013;50(5):587-92.

9. Eisenberg S, Zuk L, Carmeli E, Katz-Leurer M. Contribution of stepping while standing to function and secondary conditions among children with cerebral palsy. Pediatr Phys Ther. 2009;21(1):79-85.

10. Wright VF, Jutai JW. Evaluation of the longer-term use of the David hart Walker Orthosis by children with cerebral palsy: a 3-year prospective evaluation. Disabil Rehabil Assist Technol. 2006;1(3):155-66.
11. Kuenzle C, Brunner R. The effects of the norsk function-walking orthosis on the walking ability of children with cerebral palsy and severe gait impairment. J Prosthetics Orthotics. 2009;21(3):138-44.

12. McKeever $P$, Rossen BE, Scott $H$, Robinson-Vincent $K$, Wright $V$. The significance of uprightness: parents' reflections on children's responses to a hand-free walker for children. Disabily Soc. 2013;28(3):380-92.

13. Elo S, Kyngäs H. The qualitative content analysis process. J Adv Nurs. 2008; 62(1):107-15.

14. O'Cathain A, Thomas KJ. "Any other comments?" open questions on questionnaires - a bane or a bonus to research? BMC Med Res Methodol. 2004:4(25):1-7.

15. De Onis M. WHO motor development study: windows of achievement for six gross motor development milestones. Acta Paediatr Suppl. 2006; 95(SUPPL. 450):86-95.

16. Larkin D, Summers J. Implications of movement difficulties for social interaction, physical activity, play and sports. Developmental Motor Disorders: A Neuropsychological Perspective. The Guildford Press New York. 2004.

17. Barnes SB, Whinnery KW. Effects of functional mobility skills training for young students with physical disabilities. Except Child. 2002;68(3):313-24.

18. Koop SE. Scoliosis in cerebral palsy. Dev Med Child Neurol. 2009;51(s4):92-8

19. Balemans AC, Bolster EA, Brehm MA, Dallmeijer AJ. Physical strain: a new perspective of walking in cerebral palsy. Arch Phys Med Rehabil. 2017; 98(12):2507-13.

20. Huang I-C, Sugden D, Beveridge S. Assistive devices and cerebral palsy: the use of assistive devices at school by children with cerebral palsy. Child Care Health Dev. 2009;35(5):698-708.

21. Fowler EG, Kolobe TH, Damiano DL, Thorpe DE, Morgan DW, Brunstrom JE, Coster WJ, Henderson RC, Pitetti KH, Rimmer JH, Rose J, Stevenson RD. Promotion of physical fitness and prevention of secondary conditions for children with cerebral palsy: section on pediatrics research summit proceedings. Phys Ther. 2007;87(11):1495-510.

22. Barg CJ, Armstrong BD, Hetz SP, Latimer AE. Physical disability, stigma, and physical activity in children. Int J Disabil Dev Educ. 2010;57(4):371-82.

23. Eyssen IC, Steultjens MP, Dekker J, Terwee CB. A systematic review of instruments assessing participation: challenges in defining participation. Arch Phys Med Rehabil. 2011;92(6):983-97.

24. Badia M, Longo E, Orgaz MB, Gómez-Vela M. The influence of participation in leisure activities on quality of life in Spanish children and adolescents with cerebral palsy. Res Dev Disabil. 2013;43(9):2864-71.

25. Huang I-C, Sugden D, Beveridge S. Assistive devices and cerebral palsy: factors influencing the use of assistive devices at home by children with cerebral palsy. Child Care Health Dev. 2009;35(1):130-9.

\section{Publisher's Note}

Springer Nature remains neutral with regard to jurisdictional claims in published maps and institutional affiliations.

Ready to submit your research? Choose BMC and benefit from:

- fast, convenient online submission

- thorough peer review by experienced researchers in your field

- rapid publication on acceptance

- support for research data, including large and complex data types

- gold Open Access which fosters wider collaboration and increased citations

- maximum visibility for your research: over $100 \mathrm{M}$ website views per year

At $\mathrm{BMC}$, research is always in progress.

Learn more biomedcentral.com/submissions 